\title{
Dementia Enhances Inhibitory Actions of General Anesthetics in Hippocampal Synaptic Transmission
}

\author{
Masana Yamada, Rika Sasaki, Koki Hirota, and Mitsuaki Yamazaki \\ Department of Anesthesiology, Graduate School of Medicine and Pharmaceutical Science for Research, University of Toyama, \\ 2630 Sugitani, Toyama 930-0194, Japan \\ Correspondence should be addressed to Koki Hirota, koki@med.u-toyama.ac.jp
}

Received 12 October 2011; Accepted 1 November 2011

Academic Editor: B. Maciver

Copyright ( $) 2011$ Masana Yamada et al. This is an open access article distributed under the Creative Commons Attribution License, which permits unrestricted use, distribution, and reproduction in any medium, provided the original work is properly cited.

In order to investigate whether dementia modifies the anesthetic actions in the central nervous systems, we have studied effects of general anesthetics on the hippocampal synaptic transmission using the dementia model mice. Preliminary in vivo experiments revealed that time of loss of righting reflex following sevoflurane inhalation was more shortened in dementia mice than in healthy control mice. Field population spikes of hippocampal CA1 pyramidal neurons were elicited in vitro using orthodromic stimulation of Schaffer collateral commissural fibers (test pulse). The recurrent inhibition was enhanced with the second stimulating electrode placed in alveus hippocampi (prepulse) to activate recurrent inhibition of CA1. The prepulses were applied as train stimuli to activate release and then deplete $\gamma$-amino-butyric acid (GABA) at presynaptic terminals of inhibitory interneurons. Sevoflurane and thiopental had greater actions on inhibitory synaptic transmission in dementia model mice than in control mice. The prepulse train protocol revealed that the anesthetic-induced GABA discharge was more enhanced in dementia mice than in control mice. Dementia enhances the actions of general anesthetics due to the increase in GABA release from presynaptic terminals.

\section{Introduction}

Clinical anesthesiologists have an increasing opportunity to anesthetize patients with dementia. In order to investigate whether the dementia modifies the anesthetic actions in the central nervous systems, we have studied effects of general anesthetics on the hippocampal synaptic transmission in dementia model animals.

The senescence-accelerated mouse (SAM) has been established by Takeda [1]. There are now eight prone lines (SAM-P), and each line has a strain-specific pathological phenotype. The SAM-P8 mouse has characteristic feature of deterioration in learning and memory abilities, whereas SAM-R1 mouse has no neurological impairment (healthy control). It has been reported that amyloid $\beta$ protein deposition is observed in various regions, including hippocampus, in the brain of SAM-P8 [2]. Since the amyloid $\beta$ protein deposition is histopathological character in Alzheimer's disease [3], there is a possibility that neural disorders in SAM-P8 might be related to Alzheimer type dementia.
Previous studies from our laboratory have demonstrated that general anesthetics mainly enhance inhibitory synaptic transmission $[4,5]$ and increase neurotransmitter ( $\gamma$-aminobutyric acid, GABA) release from presynaptic terminals in hippocampus [6]. Dementia patients are associated with impairments of recent memory and learning, indicating hippocampal dysfunctions. Therefore, we hypothesized that dementia could modify the actions of general anesthetics in the central nervous system. In the present study, we studied effects of general anesthetics on the loss of righting reflex (LORR) and the synaptic transmission in hippocampal preparations from dementia model animals.

\section{Materials and Methods}

Ethical approval was obtained from the Animal Research Committee of the University of Toyama, Japan. The time for loss of righting reflex (LORR) was examined using an observable anesthetic chamber $\left(5,600 \mathrm{~cm}^{3}\right)$. Sevoflurane (3.0 vol\%) in oxygen was continuously administered to the 


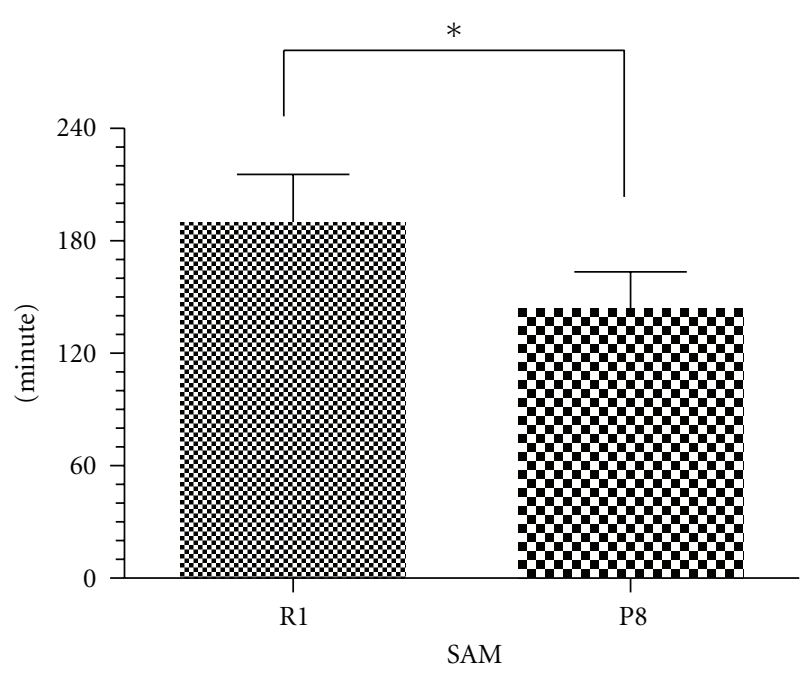

FIgURE 1: Effects of sevoflurane (3.0 vol\%) inhalation on time for loss of righting reflex (LORR) in dementia model mice (SAM-P8, $n=5$ ) and control mice (SAM-R1, $n=5$ ). Data were expressed as mean \pm SD. ${ }^{*} P=0.013$.

anesthetic chamber $(6 \mathrm{~L} / \mathrm{min})$. We put a mouse into chamber and then tilted the chamber to an angle of $45^{\circ}$ from a horizontal plane every $10 \mathrm{sec}$. LORR was determined when the mouse failed to right itself within $5 \mathrm{sec}$. Righting reflex was assessed and recorded every $10 \mathrm{sec}$.

2.1. Hippocampal Slice Preparation. Methods for the preparation of rat hippocampal slices and electrophysiological protocols have been previously described [6]. In brief, SAM mice (4 months) were deeply anesthetized with sevoflurane and then decapitated. The brain was rapidly removed, and $400 \mu \mathrm{m}$ transverse slices were prepared from the dissected hippocampus in cold, oxygenated artificial cerebrospinal fluid (ACSF) using a Rotorslicer DTY-7700 (DSK, Osaka, Japan). The slices were placed on a nylon mesh screen at the interface of ACSF liquid $(90 \mathrm{~mL} / \mathrm{h})$ and humidified $95 \% \mathrm{O}_{2} / 5 \% \mathrm{CO}_{2}$ gas $(1 \mathrm{~L} / \mathrm{min})$ phases in a recording chamber. In order to accelerate the rate of drug equilibration and to obtain stable recordings of field potentials, we have developed the liquid/gas interface brain slice chamber with minimal perfusate volume $(0.8 \mathrm{~mL})$. Slices were warmed to $37^{\circ} \mathrm{C}$ slowly and then allowed to equilibrate for 90-120 min without electrical stimulation.

2.2. Electrophysiological Recordings. A glass extracellular recording microelectrodes (3-5 M $\Omega$ filled with $2 \mathrm{~mol} / \mathrm{L}$ of $\mathrm{NaCl}$ ) was positioned in the cell body region of the CA1 pyramidal neurons to record field population spikes (PSs). A bipolar stimulating electrode (tungsten steel, coated with epoxy resin, Unique Medical, Tokyo, Japan) was placed in the region of the Schaffer-collateral-commissural fibers (Sch) to stimulate the input to CA1 neurons, and a second electrode was located in the region of the alveus hippocampi (Alv) to activate inhibitory interneurons of the CA1 (Figure 1) [6]. PS amplitudes were determined from peak positive to peak negative of the waveform. In order to establish the recurrent inhibition enhanced (RIE) circuit, the Alv was stimulated with a prepulse to activate GABA-mediated recurrent inhibition. Electrical stimulation of the Sch was then applied (10 $\mathrm{ms}$ after the prepulse) as a test pulse to elicit PS. When the recurrent inhibition was enhanced via presynaptic mechanisms, the reduction of PS was detected [4]. A number of prepulses $(n=0--320)$ were applied as a train $(200 \mathrm{~Hz})$ in order to access the GABA release from presynaptic terminals (prepulse train protocol). DL-2amino-5-phosphonovaleric acid (AP5, $10^{-4} \mathrm{~mol} / \mathrm{L}$ ) was used to prevent NMDA (N-methyl-D-aspartate) receptor-related synaptic plasticity [6].

Square-wave stimuli (5-10 volt, $50 \mu$ s), generated with an SEN-3301 stimulator (Nihon Kohden, Tokyo, Japan), were delivered to both pathways (Sch and Alv) simultaneously. The minimal stimulus intensity that elicited the maximal amplitude (maximal stimulus) was normally used. Stimulus frequency was fixed at $0.03 \mathrm{~Hz}$, since the input frequency can modify anesthetic actions [6]. Field potentials were amplified with an MEZ-8301 amplifier (Nihon Kohden, Tokyo, Japan) and filtered $1 \mathrm{~Hz}-10 \mathrm{kHz}$. Analog-digital conversions of data were made at a rate of $100 \mathrm{kHz}$ using an InstruNet (GW, Somerville, MA). The results were stored on the hard drive of a Macintosh computer (Apple, Cupertino, CA), and PS amplitudes and EPSP slopes were analyzed using SuperScope software (GW, Somerville, MA).

2.3. Drug Application and Data Acquisition. All preparations used in the present study showed control variability $<5 \%$ during the initial data acquisition period and following washout of anesthetic drugs. Recovery responses were recorded at least $30 \mathrm{~min}$ after washout of anesthetic-equilibrated ACSF from the chamber. Sevoflurane was applied as vapors in the prewarmed carrier gas $\left(95 \% \mathrm{O}_{2} / 5 \% \mathrm{CO}_{2}\right)$ above the slices using a calibrated commercial vaporizer ( $\mathrm{Tec} 3$, Omeda, Steeton, West Yorkshire, UK). Concentrations, expressed as volume percent (vol\%), refer to the dial settings on the vaporizer. Concentrations of sevoflurane in the perfusate of the recording chamber were determined using a portable volatile gas analyzer (OSP, Saitama, Japan): a linear relationship $\left(6.5 \times 10^{-4} \mathrm{~mol} / \mathrm{L}\right.$ per $1.0 \mathrm{vol} \%$ ) up to $5.0 \mathrm{vol} \%$.

Thiopental was dissolved in ACSF at required concentrations prior to use. All anesthetics and drugs were applied for a minimum of $20 \mathrm{~min}$ to reach equilibrium, as previously demonstrated. Since $\mathrm{ED}_{50}$ s have been determined previously $[4,5], 3.0 \mathrm{vol} \%$ of sevoflurane and $10^{-5} \mathrm{~mol} / \mathrm{L}$ of thiopental were used in the present study. The composition of the ACSF was (mmol/L): $\mathrm{NaCl} 124, \mathrm{KCl} 5, \mathrm{CaCl}_{2} 2, \mathrm{NaH}_{2} \mathrm{PO}_{4}$, $1.25, \mathrm{MgSO}_{4} 2, \mathrm{NaHCO}_{3} 26, \mathrm{AP} 5 \mathrm{0.1}$, and glucose 10, prepared with purified water. The ACSF was precooled (8$10^{\circ} \mathrm{C}$ ) and kept saturated with $95 \% \mathrm{O}_{2} / 5 \% \mathrm{CO}_{2}$ gas mixture before use ( $\mathrm{pH}$ 7.1-7.3). Sevoflurane was purchased from Maruishi Pharmaceutical Co. (Osaka, Japan). Thiopental was purchased from Tanabe Pharmaceutical Co. (Osaka, Japan). All of the other chemicals used were purchased from Sigma (St. Louis, MO). 


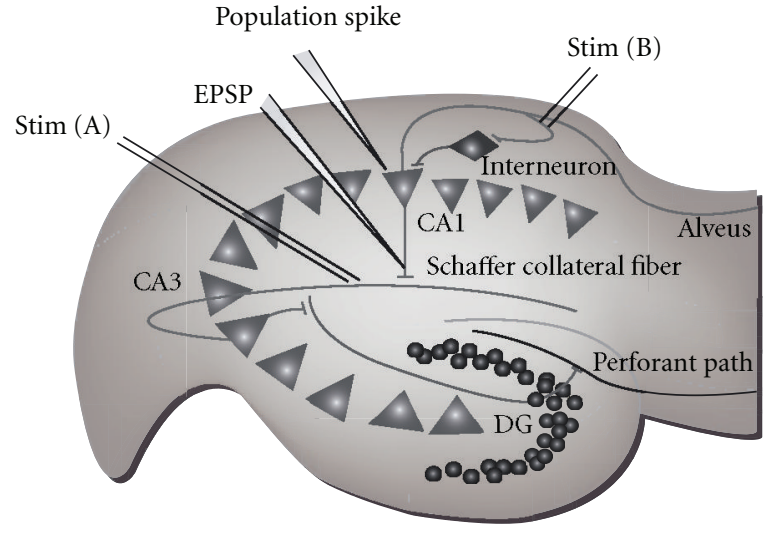

(a)

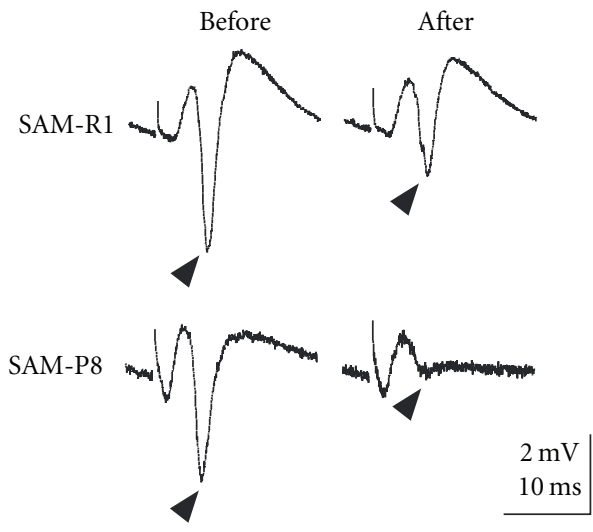

(b)

FIGURE 2: (a) A glass extracellular recording microelectrodes for recording population spike was placed in the cell body of CA1 pyramidal neurons. A bipolar stimulating electrode (for "test pulse") was located in the region of Schaffer-collateral-commissural fibers to stimulate the input to CA1 neurons, and a second electrode (for "prepulse") was placed in the region of alveus hippocampi in order to establish the recurrent inhibition enhanced (RIE) circuit. DG: dentate gyrus. Stim: stimulus electrode. (b) Representative recordings of effects of sevoflurane (3.0 vol\%) on the field population spikes of RIE circuit in dementia model mice (SAM-P8) and control mice (SAM-R1). Triangles indicate peak amplitude of the population spikes.

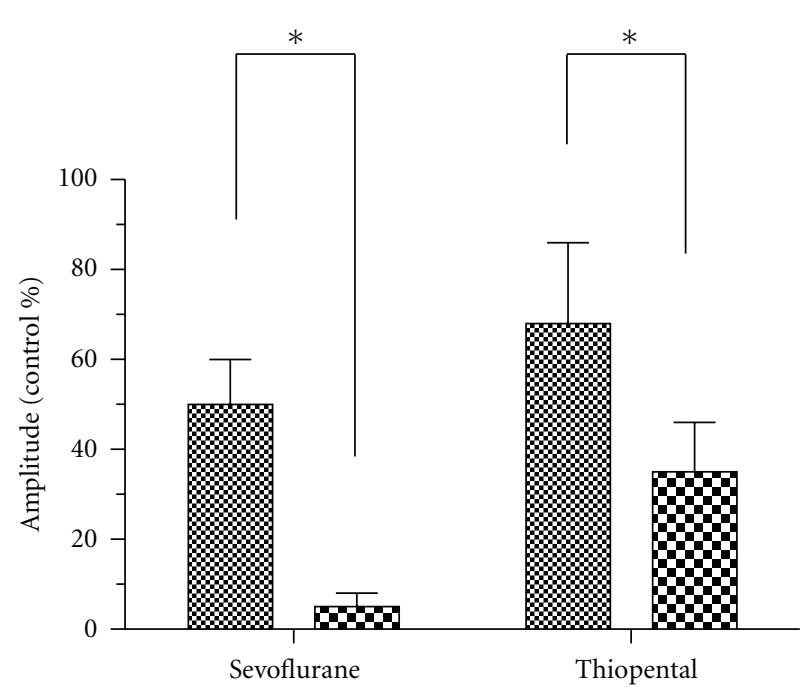

\%AM R1

D. SAM P8

FIGURE 3: Effects of sevoflurane $(3.0 \mathrm{vol} \%)$ and thiopental $\left(10^{-5} \mathrm{~mol} / \mathrm{L}\right)$ on the peak amplitude of population spike in the recurrent inhibition enhanced (RIE) circuit in dementia model mice (SAM-P8, $n=5$ ) and control mice (SAM-R1, $n=5)$. Data were expressed as $\%$ of control (mean $\pm \mathrm{SD}$ ). ${ }^{*} P<0.05$.

2.4. Statistical Analysis. Numerical data were expressed as mean \pm SD. Statistical differences were tested by repeatedmeasures analysis of variance (ANOVA), and differences between paired sets of data were compared by the Bonferroni/Dunn test. Differences between two groups were tested by Student's $t$-test. A $P$ value of $<0.05$ was considered significantly different. Statistical analysis was performed using Prism software (GraphPad, San Diego, CA).

\section{Results}

3.1. Time for LORR in Dementia Model Mice and Control Mice. In the presence of $3.0 \mathrm{vol} \%$ sevoflurane, the righting reflex of control mice (SAM-R1) disappeared in $190 \pm 11 \mathrm{sec}$ (Figure 1). The time for LORR was significantly shortened (to $76 \%$ of SAM-R1) in dementia model mice (SAM-P8). The different sensitivity to sevoflurane between dementia and control mice in vivo prompted us to investigate the following electrophysiological mechanisms in hippocampal synaptic transmission in vitro.

3.2. Field Population Spikes of RIE Circuits in Hippocampal Slices. We employed the GABA-mediated recurrent inhibitory pathway using the RIE circuits in SAM-R1 and P8 mice to study the effects of thiopental and sevoflurane (Figure 2(a)). The representative recordings of effects of sevoflurane in hippocampal RIE circuits were shown in Figure 2(b). Both sevoflurane (3.0 vol\%) and thiopental $\left(10^{-5} \mathrm{~mol} / \mathrm{L}\right)$ reduced the amplitude of PS, and the inhibitory actions were more prominent in dementia model compared to control (Figure 3).

3.3. Neurotransmitter (GABA) Release from Presynaptic Terminals. Since we hypothesized that the dementia-related sensitivity to general anesthetics could be due to an enhancement in recurrent inhibition, we examined neurotransmitter (GABA) release from presynaptic terminals using the prepulse protocol [6]. As shown in the inset of Figure 4, the prepulse train protocol initially enhances release of GABA from presynaptic terminals (phase I: reduction in the test-pulse amplitude), and then induces a depletion of neurotransmitter (phase II: disinhibition of the amplitude).

In the absence of anesthetic (i.e., before anesthetic application), the duration of GABA release was longer in SAMP8 compared to SAM-R1. In the presence of sevoflurane 
SAM-R1

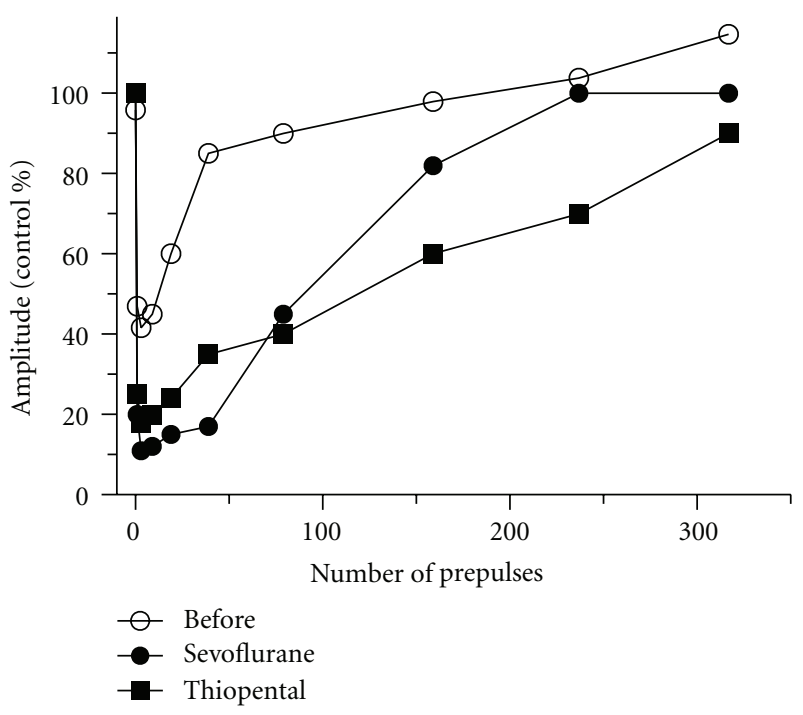

(a)

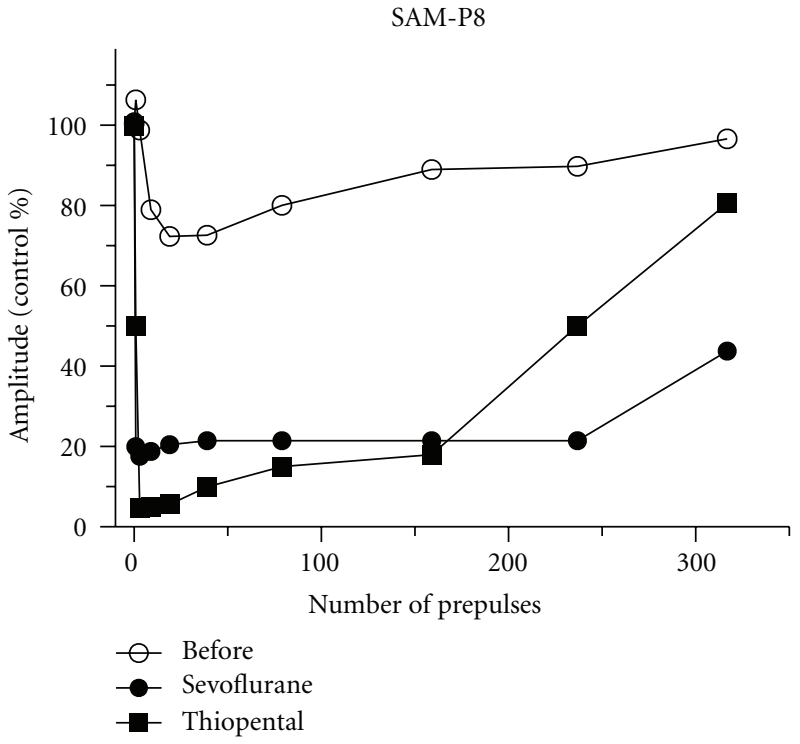

(b)

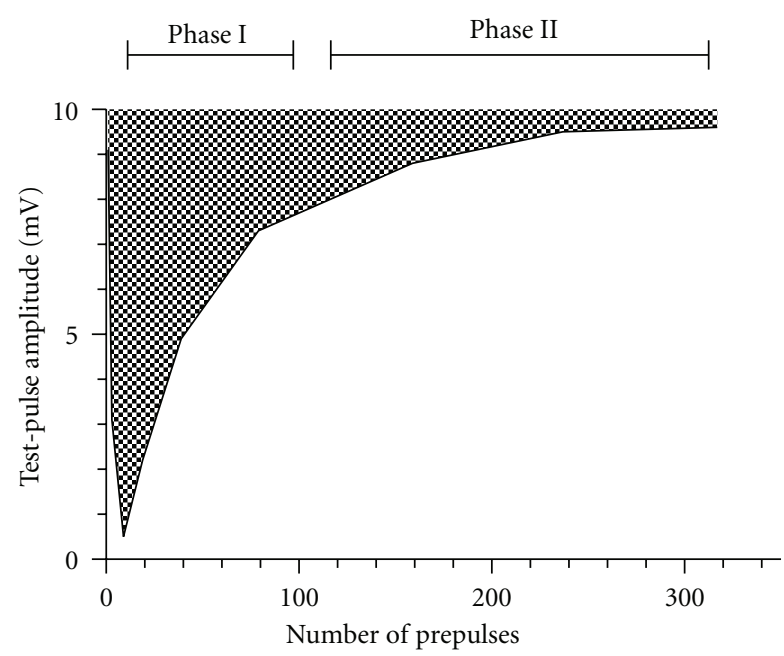

(c)

FIGURE 4: The prepulse train protocol was employed to examine the $\gamma$-amino-butyric acid (GABA) release from presynaptic terminals of inhibitory interneurons. Different numbers of prepulses (0-320) were applied at $200 \mathrm{~Hz}$ to activate GABA release. The relationships between number of prepulses and population spike amplitudes of dementia model mice (SAM-P8, $n=5)$ and control mice (SAM-R1, $n=5)$ in the absence and presence of sevoflurane $(3.0 \mathrm{vol} \%)$ or thiopental $\left(10^{-5} \mathrm{~mol} / \mathrm{L}\right)$ are shown. Inset. The prepulse train protocol initially enhances release of GABA from presynaptic terminals (phase I: reduction in the test-pulse amplitude) and then induces a depletion of neurotransmitter (phase II: disinhibition of the amplitude).

$(3.0 \mathrm{vol} \%)$ or thiopental $\left(10^{-5} \mathrm{~mol} / \mathrm{L}\right)$, GABA release was enhanced; the effects were greater in SAM-P8 compared to SAM-R1. Therefore, the difference in the kinetics of neurotransmitter (GABA) release from presynaptic terminals in dementia and control animals may be responsible for the observed dementia-related sensitivity to general anesthetics.

\section{Discussion}

To our knowledge, this is the first report of effects of dementia on actions of general anesthetics in vivo and in vitro. The time for LORR following general anesthetic application was significantly shortened in dementia mice compared with control mice. The corresponding electrophysiological studies revealed that inhibitory actions of general anesthetics on hippocampal synaptic transmission were more enhanced in dementia model animals.

In order to assess mechanisms for the dementia-related modification of general anesthetic actions, we utilized the prepulse train protocol to accelerate the neurotransmitter (GABA) release. The prepulse train protocol was developed in our laboratory [6]. In hippocampal neurons, a singleaction potential releases $0.5 \%$ of neurotransmitter pool from presynaptic terminals [7]. Therefore, the prepulse train could first enhance GABA release and attenuate the PS amplitude (phase I). Subsequently, more than 200 pulses of a prepulse 
train could release all the active pool of GABA and temporally deplete readily releasable neurotransmitter (phase II). General anesthetics depressed phase I and prolonged the onset of phase II, since general anesthetics induce the GABA release from presynaptic terminals [6]. The present study demonstrated that dementia enhanced the depression of phase I and prolongation of phase II, suggesting that dementia increases anesthetic-induced GABA release at presynaptic terminals.

Recent advances in neuroscience have differentiated dementia from physiological aging. Aging itself is known to increase the excitatory synaptic transmission in the central nervous systems [8], whereas dementia compounds the neurodegenerative disorders on neurons [9]. For example, the amyloid $\beta$ protein in Alzheimer's disease has been known to sensitize neurons to excitotoxic death, resulting in imbalance of synaptic transmission [10]. The selective dysfunction in alpha5-subunit of $\mathrm{GABA}_{\mathrm{A}}$ receptor has been implicated in cognitive dysfunctions [11], since reduction in $\alpha 5$-subunit of $\mathrm{GABA}_{\mathrm{A}}$ receptor function is proposed as a cognition enhancement for senile dementia $[12,13]$. Interestingly, the $\alpha 5-\mathrm{GABA}_{\mathrm{A}}$ receptor has been reported to be extremely sensitive to general anesthetics [14]. Taken together, dementia modifies the inhibitory synaptic transmission in the central nervous systems and can interfere with anesthetic potency.

In conclusion, our results first demonstrated that dementia enhanced inhibitory actions of general anesthetics in vivo and in vitro. The modification of presynaptic GABA release can explain the dementia-induced enhancements of general anesthetic actions. Present findings would provide basic evidences for clinical anesthesia of patients with dementia.

\section{Acknowledgments}

The authors are extremely grateful to Professor Sheldon H. Roth, University of Calgary, Alberta, Canada, for many helpful discussions, suggestions on this paper, and the courtesy of providing the brain slice chambers. This study was supported by a grant from the Ministry of Education, Culture, Sports, Science and Technology (MEXT), Japan, Grant-in-Aid for Scienctific Research (C) 22591704.

\section{References}

[1] T. Takeda, "Senescence-accelerated mouse (SAM): a biogerontological resource in aging research," Neurobiology of Aging, vol. 20, no. 2, pp. 105-110, 1999.

[2] M. Takemura, S. Nakamura, I. Akiguchi et al., " $\beta / A 4$ proteinlike immunoreactive granular structures in the brain of senescence-accelerated mouse," American Journal of Pathology, vol. 142, no. 6, pp. 1887-1897, 1993.

[3] L. M. Ittner and J. Götz, "Amyloid- $\beta$ and tau-a toxic pas de deux in Alzheimer's disease," Nature Reviews Neuroscience, vol. 12, pp. 67-72, 2011.

[4] T. Asahi, K. Hirota, R. Sasaki, Y. Mitsuaki, and S. H. Roth, "Intravenous anesthetics are more effective than volatile anesthetics on inhibitory pathways in rat hippocampal CA1," Anesthesia and Analgesia, vol. 102, no. 3, pp. 772-778, 2006.
[5] M. Wakasugi, K. Hirota, S. H. Roth, and Y. Ito, "The effects of general anesthetics on excitatory and inhibitory synaptic transmission in area CA1 of the rat hippocampus in vitro," Anesthesia and Analgesia, vol. 88, no. 3, pp. 676-680, 1999.

[6] K. Hirota, R. Sasaki, S. H. Roth, and M. Yamazaki, "Presynaptic actions of general anesthetics are responsible for frequencydependent modification of synaptic transmission in the rat hippocampal CA1," Anesthesia and Analgesia, vol. 110, no. 6, pp. 1607-1613, 2010.

[7] T. A. Ryan and S. J. Smith, "Vesicle pool mobilization during action potential firing at hippocampal synapses," Neuron, vol. 14, no. 5, pp. 983-989, 1995.

[8] C. A. Barnes, G. Rao, and B. L. McNaughton, "Increased electrotonic coupling in aged rat hippocampus: a possible mechanism for cellular excitability changes," Journal of Comparative Neurology, vol. 259, no. 4, pp. 549-558, 1987.

[9] M. P. Mattson and T. Magnus, "Ageing and neuronal vulnerability," Nature Reviews Neuroscience, vol. 7, no. 4, pp. 278-294, 2006.

[10] M. P. Mattson, "Pathways towards and away from Alzheimer's disease," Nature, vol. 430, no. 7000, pp. 631-639, 2004.

[11] D. J. Nutt, M. Besson, S. J. Wilson, G. R. Dawson, and A. R. Lingford-Hughes, "Blockade of alcohol's amnestic activity in humans by an $\alpha 5$ subtype benzodiazepine receptor inverse agonist," Neuropharmacology, vol. 53, no. 7, pp. 810-820, 2007.

[12] N. Collinson, F. M. Kuenzi, W. Jarolimek et al., "Enhanced learning and memory and altered GABAergic synaptic transmission in mice lacking the $\alpha 5$ subunit of the $\mathrm{GABA}_{\mathrm{A}}$ receptor," Journal of Neuroscience, vol. 22, no. 13, pp. 5572-5580, 2002.

[13] J. R. Atack, "Preclinical and clinical pharmacology of the $\mathrm{GABA}_{\mathrm{A}}$ receptor $\alpha 5$ subtype-selective inverse agonist $\alpha 5 \mathrm{IA}$," Pharmacology and Therapeutics, vol. 125, no. 1, pp. 11-26, 2010.

[14] V. Y. Cheng, L. J. Martin, E. M. Elliott et al., " $\alpha 5 \mathrm{GABA}_{\mathrm{A}}$ receptors mediate the amnestic but not sedative-hypnotic effects of the general anesthetic etomidate," Journal of Neuroscience, vol. 26, no. 14, pp. 3713-3720, 2006. 


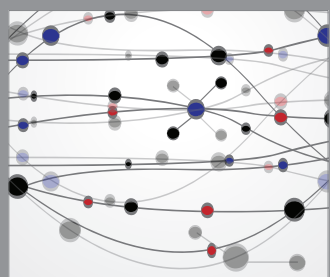

The Scientific World Journal
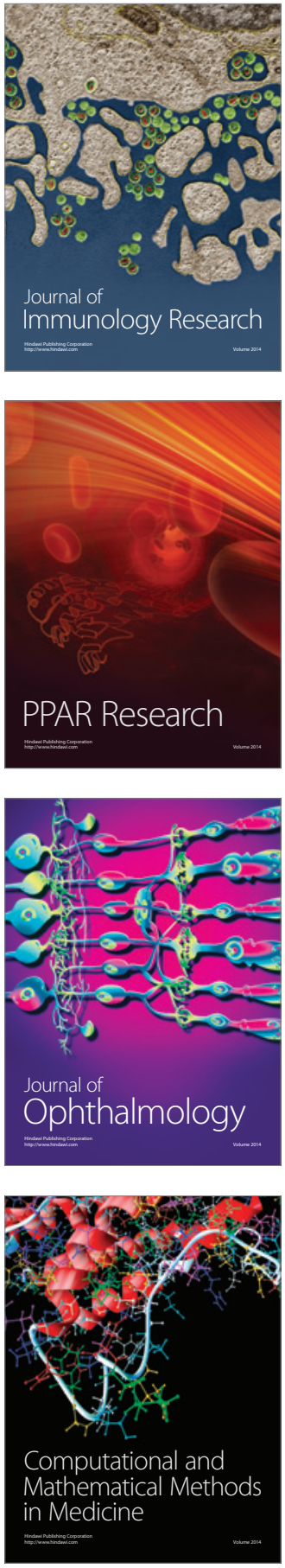

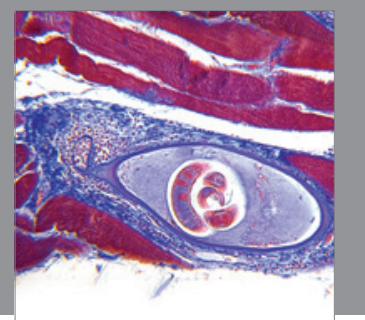

Gastroenterology

Research and Practice
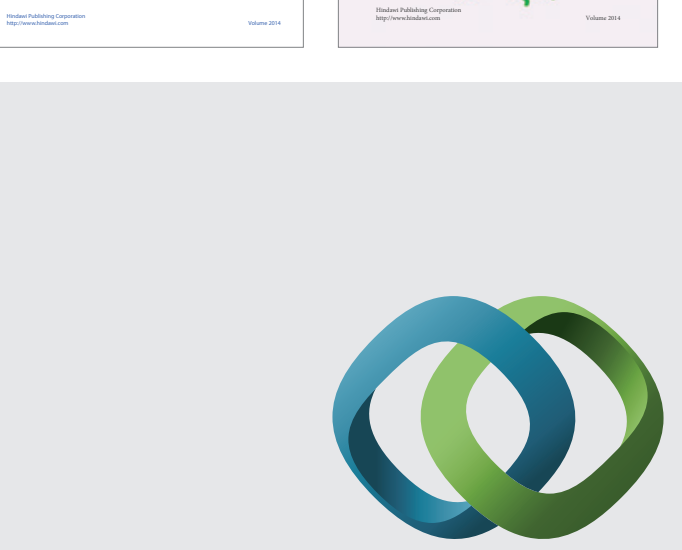

\section{Hindawi}

Submit your manuscripts at

http://www.hindawi.com
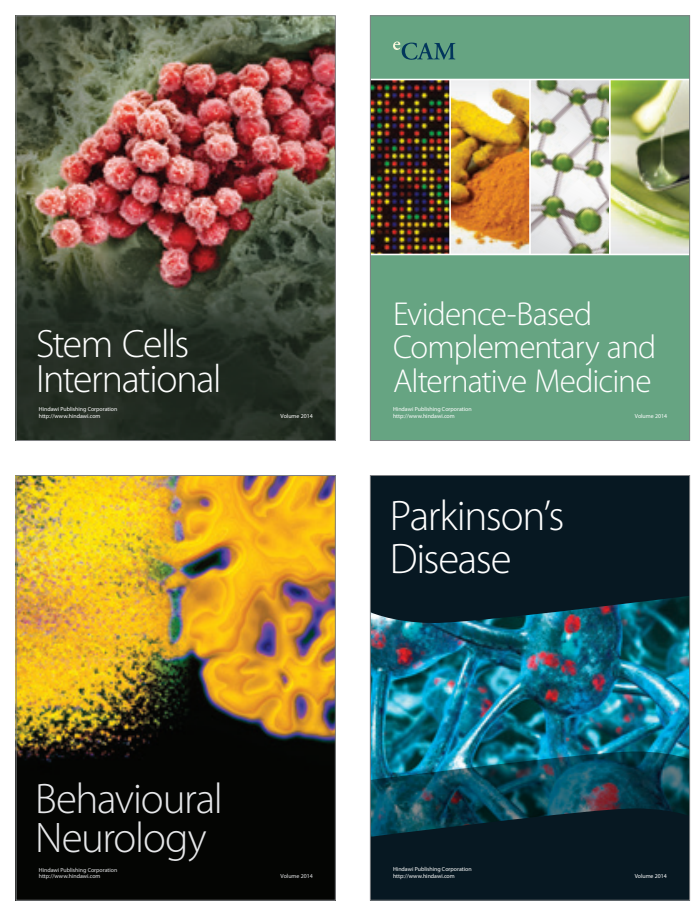

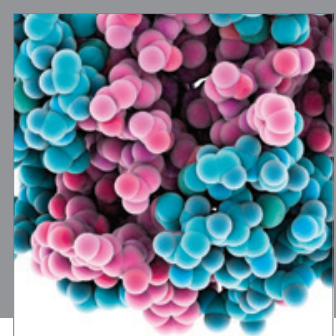

Journal of
Diabetes Research

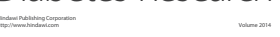

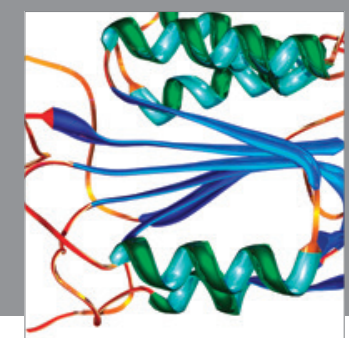

Disease Markers
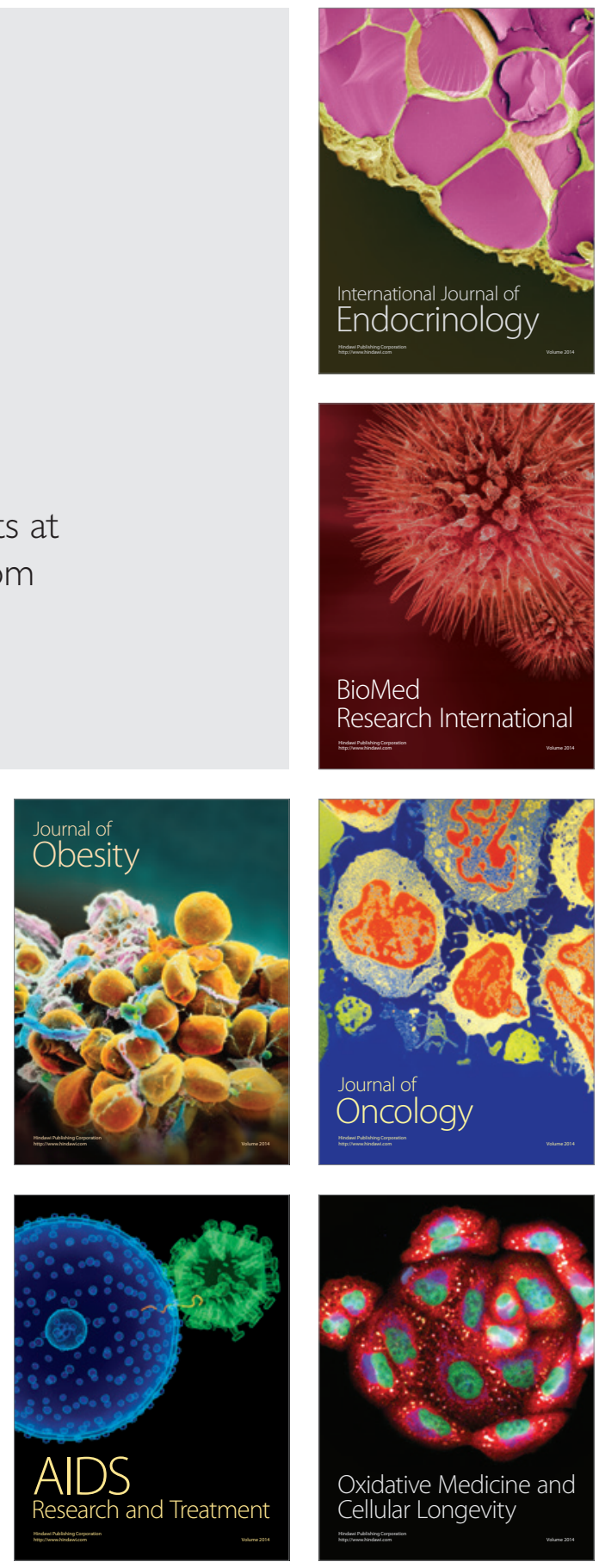